\section{Association of circulating CXCL10 and CXCL11 with systemic sclerosis}

Systemic sclerosis (SSc) is an autoimmune disorder defined 'vascular' disease ${ }^{1}$ due to early defective angiogenesis ending in severe multiorgan fibrosis. ${ }^{2}$ Biomarker(s) mirroring early microvascular derangements in SSc, ${ }^{3}$ potentially useful for very early diagnosis, are lacking.

C-X-C angiostatic chemokines induced by interferon- $\gamma$, like CXCL10 and CXCL11, are involved in vasculopathy and associate with more severe SSc. ${ }^{45}$ We investigated whether the shift from very early diagnosis of SSc (VEDOSS), when vasculopathy and fibrosis are at very low degree, to definite SSc elicits serum CXCL10/CXCL11 modifications.

CXCL10/CXCL11 were measured by multiplatform bead array in 26 healthy subjects; 62 sera from women admitted to the Scleroderma Clinic of Policlinico Umberto I, Sapienza University of Rome: 34 sera were from VEDOSS ${ }^{6}$ (mean age $50.50 \pm 13.66$ years, mean disease duration $11.1 \pm 4.2$ months) and 28 sera from patients with SSc (mean age $56.56 \pm 12.45$ years, mean disease $91.3 \pm 11.73$ months) fulfilling the new American College of Rheumatology/European League Against Rheumatism 2013 classification $^{7}$; table 1 reports patient characteristics.

Within VEDOSS, 29 subjects had a second blood sample collected during follow-up (T1, 40.67 \pm 5.46 months); for each one, we compared baseline (T0) and T1 serum chemokines. Informed consent was obtained.

Table 1 Demographic and clinical characteristics of patients with very early diagnosis of systemic sclerosis (VEDOSS) and systemic sclerosis (SSc) (whose sera sample collection was used in the study).

\begin{tabular}{lcl}
\hline Characteristics & SSc $(\mathbf{n}=\mathbf{2 8})$ & VEDOSS $(\mathbf{n}=\mathbf{3 4})$ \\
\hline Age (years) & $56.56 \pm 12.45$ & $50.50 \pm 13.66$ \\
Male & - & - \\
Female & 28 & 34 \\
IcSSc subsets & $25(89 \%)$ & - \\
dcSSc subsets & $3(11 \%)$ & - \\
Disease duration (months) & $91.3 \pm 11.73$ & $11.1 \pm 4.2$ \\
ANA & $26(93 \%)$ & $31(91 \%)$ \\
ACA & $16(57 \%)$ & $17(50 \%)$ \\
Anti-topoisomerase I (Scl-70) & $4(14 \%)$ & $5(15 \%)$ \\
Digital ulcers & $4(14 \%)$ & - \\
Normal NVC pattern & $1(4 \%)$ & $13(38 \%)$ \\
Early NVC pattern & $6(21 \%)$ & $11(32 \%)$ \\
Active NVC pattern & $13(46 \%)$ & $9(26 \%)$ \\
Late NVC pattern & $8(29 \%)$ & $1(3 \%)$ \\
Interstitial lung disease & $6(21 \%)$ & - \\
\hline Ag and diseas &
\end{tabular}

Age and disease duration were expressed as mean $\pm \mathrm{SD} / \mathrm{SE}$; disease duration was calculated since the first Raynaud phenomenon in VEDOSS and non-Raynaud symptom of SSc. Patients were not receiving corticosteroids, immunosuppressant or other disease-modifying drugs; patients with cardiac disease, pulmonary artery hypertension or active diseases other than SSc were excluded. Interstitial lung disease was determined by high-resolution computed tomography CT scan. ACA, anti-centromere antibodies; ANA, antinuclear antibodies; dcSSc, diffuse cutaneous SSc; ICSSc, limited cutaneous SSC; NVC, nailfold videocapillaroscopy.
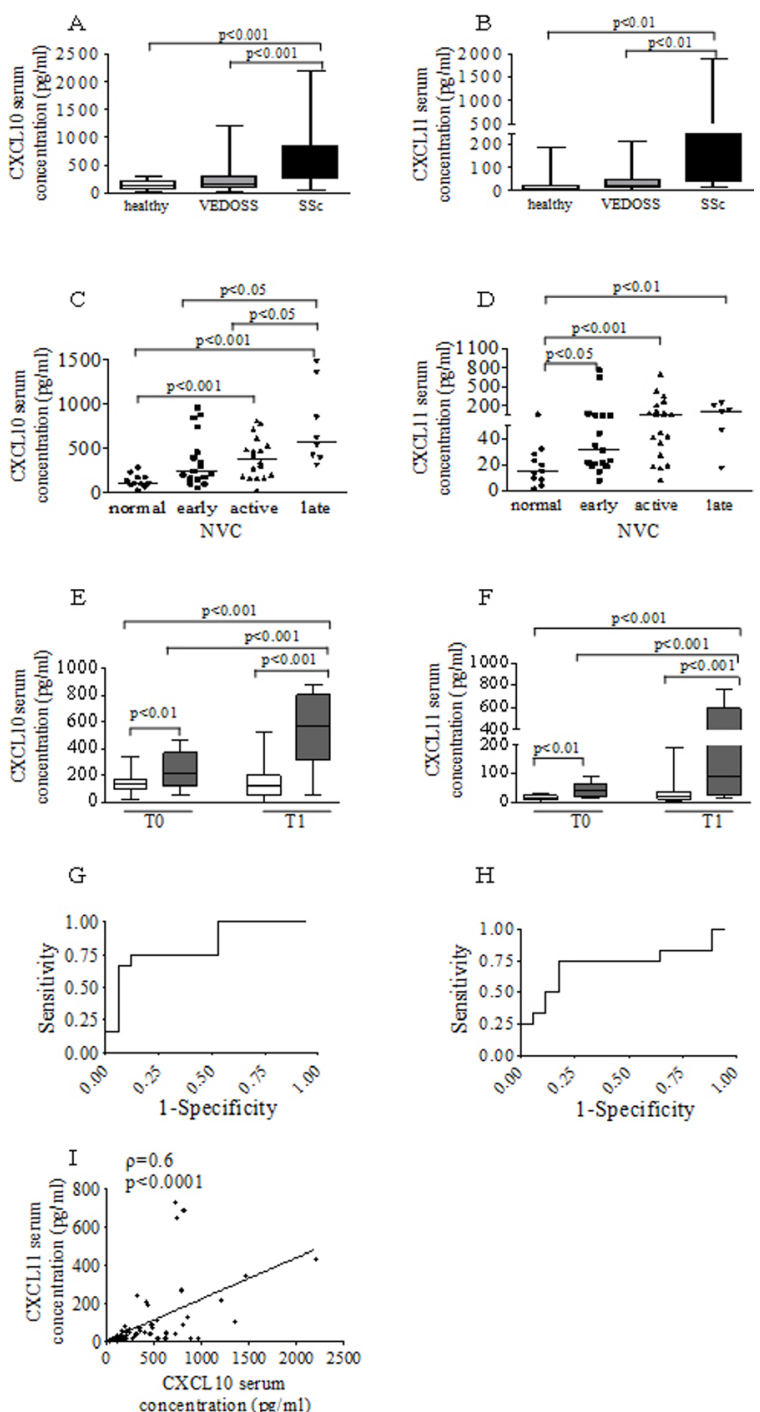

Figure 1 CXCL10 and CXCL11 serum-level modifications associated with very early diagnosis of systemic sclerosis (VEDOSS) and systemic sclerosis (SSC) condition. Baseline serum CXCL10 and CXCL11 levels in VEDOSS versus SSC and healthy subjects (A, B); CXCL10 and CXCL11 serum concentration according to capillaroscopic patterns in VEDOSS and SSC (C, D) - normal, early, active and late; CXCL10 and CXCL11 baseline (T0) and follow-up (T1) determination in sera of VEDOSS subjects shifted (grey boxes) or not (empty boxes) to SSc (E, F); receiver operating characteristic (ROC) curves constructed using chemokine baseline levels (T0) to identify CXCL10 and CXCL11 threshold (cut-off value) capable of discriminating VEDOSS subjects shifting or not to SSc (G, H); CXCL10 and CXCL11 serum-level correlation (I). GraphPad Prism V.5 software (GraphPad Software, La Jolla, California, USA) and SPSS V.24.0 software (SPSS) were used for statistical analysis. The Kolmogorov-Smirnov test was used for normal distribution of the data. Groupwise comparisons were performed using the MannWhitney $\mathrm{U}$ test. ROC curves were to give a graphic representation of the relationship between true-positive fraction (sensitivity, SE) and false-positive fraction (1 - specificity, Sp); ROC curves were assessed by plotting the values of $1-\mathrm{Sp}$ against $\mathrm{Se}$ in a squared box, where the ROC's area under the curve (AUC) is used to measure the performance of a diagnostic test. The AUC lies in the interval $0.5-1.0$, so that the greater the area, the better the performance of the variable being examined. Chemokine association was determined using Pearson's correlation coefficient. For all tests, a two-sided $p$ value $<0.05$ was considered significant. NVC, nailfold videocapillaroscopy. 
CXCL10/CXCL11 were significantly higher in SSc (median: CXCL10 497.70 (range: 48.49-2206) pg/mL; CXCL11 107.20 (15.00-882) pg/mL) versus VEDOSS (CXCL10 168.70 (21.661202) pg/mL; CXCL11 23.30 (1.47-217.20) pg/mL) or healthy subjects (CXCL10 $145.40 \quad(15.86-310.30) \mathrm{pg} / \mathrm{mL}$; CXCL11 $10.90(4.30-189.20) \mathrm{pg} / \mathrm{mL}$ ) (figure 1A, B). All patients showed serum chemokines stratification according to capillaroscopic patterns (figure 1C, D), while chemokine levels did not significantly differ when related to anti-centromere antibodies or anti-topoisomerase positivity (not shown). VEDOSS subsequently shifted to SSc showed higher baseline chemokines (T0: CXCL10 217 (53.68-469.00)pg/mL; CXCL11 40.57 (15.74-92.04) pg/mL) versus subjects persistent in VEDOSS condition (T0: CXCL10 137.70 (21.66-339) pg/mL; CXCL11 $17.47(1.47-32.03) \mathrm{pg} / \mathrm{mL}$ ) (figure 1E, F). Only VEDOSS shifted to SSc showed CXCL10/CXCL11 increase at T1 (570.80 (53.25-875.20) pg/mL). Both chemokines were able to discriminate VEDOSS subjects developing SSc (figure 1G, H) with the following cut-off values, identified by receiver operating characteristic (ROC) analysis: CXCL10 $\geq 165 \mathrm{pg} / \mathrm{mL}$, area under the curve $(\mathrm{AUC})=0.70$ (95\% CI 0.52 to $0.94, \mathrm{p}<0.01)$ with 0.75 sensitivity and 0.70 specificity; CXCL11 $\geq 29.67 \mathrm{pg} / \mathrm{mL}$, $\mathrm{AUC}=0.80$ (95\% CI 0.67 to $0.99, \mathrm{p}<0.01)$ with 0.75 sensitivity and 0.88 specificity. Serum CXCL10 and CXCL11 positively correlated $(\rho=0.6, p<0.0001)$ (figure 1I).

Due to CXCL10/CXCL11 detrimental effects on vessel homeostasis, ${ }^{589}$ their higher baseline concentration in VEDOSS thereafter developing SSc likely mirrors the earliest vascular bed alteration(s)/modification(s)/rearrangement(s) occurring when vasculopathy is still at low degree. With vascular damage progression, serum chemokines increased, as suggested by level stratification according to the capillaroscopic patterns. VEDOSS subjects retaining lower CXCL10/CXCL11 overtime (at T0 and T1) did not evolve to definite SSc and maintained normal capillaroscopic pattern.

The small sample size investigated did not allow more robust combined analysis, completed by several validation stages, required by studies on functional biomarkers. ${ }^{10}$ Result confirmation needs larger sample size, including more clinical variables and closer follow-up. This preliminary cross-sectional/retrospective analysis encourages further and larger works to ascertain if chemokines may be really the turning point from VEDOSS to definite SSc.

Clara Crescioli, ${ }^{1}$ Clarissa Corinaldesi, ${ }^{1,2}$ Valeria Riccieri, ${ }^{3}$ Valeria Raparelli, ${ }^{4}$ Massimiliano Vasile, ${ }^{3}$ Francesco Del Galdo, ${ }^{2,5}$ Guido Valesini, ${ }^{3}$

Andrea Lenzi, ${ }^{4}$ Stefania Basili, ${ }^{3}$ Cristina Antinozzi ${ }^{1}$

1 Department of Movement, Human and Health Sciences, University of Rome "Foro Italico", Rome, Italy

${ }^{2}$ Leeds Institute of Rheumatic and Musculoskeletal Medicine, University of Leeds, Leeds, UK

${ }^{3}$ Department of Internal Medicine and Medical Specialties, Sapienza University of Rome, Rome, Italy

${ }^{4}$ Department of Experimental Medicine, Sapienza University of Rome, Rome, Italy ${ }^{5}$ Scleroderma Programme, NIHR Leeds Musculoskeletal Biomedical Research Centre, Leeds, UK

Correspondence to Professor Clara Crescioli, Department of Movement, Human and Health Sciences, Section of Health Sciences, Università degli Studi di Roma "Foro Italico", Rome 00135, Italy; clara.crescioli@uniroma4.it

Handling editor Josef S Smolen
Acknowledgements The authors thank Dr Silvia Giannattasio, University of Rome "Foro Italico", for her support and assistance.

Collaborators Dr Silvia Giannattasio.

Contributors All authors were involved in the article drafting and crtitical revisions of important intellectual and experimental contents.All authors approved the final version to be published.

Funding This research was supported by the grant Scientific Independence of young Researchers (SIR) (protocol no. RBSI14D5NX).

Competing interests None declared.

Patient consent Obtained.

Ethics approval Sapienza University of Rome Ethic Committee (protocol no. 2567/15).

Provenance and peer review Not commissioned; externally peer reviewed.

\section{6 OPEN ACCESS}

Open access This is an open access article distributed in accordance with the Creative Commons Attribution Non Commercial (CC BY-NC 4.0) license, which permits others to distribute, remix, adapt, build upon this work non-commercially, and license their derivative works on different terms, provided the original work is properly cited and the use is non-commercial. See: http://creativecommons.org/ licenses/by-nc/4.0/

(c) Article author(s) (or their employer(s) unless otherwise stated in the text of the article) 2018. All rights reserved. No commercial use is permitted unless otherwise expressly granted.

$S B$ and $C A$ contributed equally.

SB and CA share the last co-authorship.

\section{Check for updates}

To cite Crescioli C, Corinaldesi C, Riccieri V, et al. Ann Rheum Dis 2018;77:1845-1846.

Received 16 February 2018

Revised 12 April 2018

Accepted 20 April 2018

Published Online First 14 May 2018

Ann Rheum Dis 2018;77:1845-1846. doi:10.1136/annrheumdis-2018-213257

\section{REFERENCES}

1 Matucci-Cerinic M, Kahaleh B, Wigley FM. Review: evidence that systemic sclerosis is a vascular disease. Arthritis Rheum 2013;65:1953-62.

2 Varga J, Abraham D. Systemic sclerosis: a prototypic multisystem fibrotic disorder. J Clin Invest 2007;117:557-67.

3 Chora I, Romano E, Manetti M, et al. Evidence for a derangement of the microvascular system in patients with a very early diagnosis of systemic sclerosis. J Rheumatol 2017:44:1190-7.

4 Bellando Randone S, George J, Mazzotta C, et al. Angiostatic and Angiogenic Chemokines in Systemic Sclerosis: An Overview. J Scleroderma Relat Disord 2017;2:1-10.

5 Liu X, Mayes MD, Tan FK, et al. Correlation of interferon-inducible chemokine plasma levels with disease severity in systemic sclerosis. Arthritis Rheum 2013;65:226-35.

6 Avouac J, Fransen J, Walker UA, et al. Preliminary criteria for the very early diagnosis of systemic sclerosis: results of a Delphi Consensus Study from EULAR Scleroderma Trials and Research Group. Ann Rheum Dis 2011:70:476-81.

7 van den Hoogen F, Khanna D, Fransen J, et al. 2013 classification criteria for systemic sclerosis: an American college of rheumatology/European league against rheumatism collaborative initiative. Ann Rheum Dis 2013;72:1747-55.

8 Christmann RB, Hayes E, Pendergrass S, et al. Interferon and alternative activation of monocyte/macrophages in systemic sclerosis-associated pulmonary arterial hypertension. Arthritis Rheum 2011;63:1718-28.

9 Mach F, Sauty A, larossi AS, et al. Differential expression of three T lymphocyte-activating CXC chemokines by human atheroma-associated cells. J Clin Invest 1999;104:1041-50 